\title{
Multiple-Particle Nanoantennas for Enormous Enhancement and Polarization Control of Light Emission
}

\author{
Zhipeng Li, ${ }^{\dagger}$ Timur Shegai, ${ }^{\ddagger}$ Gilad Haran, ${ }^{*}$ and Hongxing $\mathrm{Xu}^{\mathrm{t}, \varsigma_{\text {.* }}}$ \\ ${ }^{\dagger}$ Beijing National Laboratory for Condensed Matter Physics, Institute of Physics, Chinese Academy of Sciences, Beijing 100190, China, ${ }^{\ddagger}$ Department of Chemical Physics, \\ Weizmann Institute of Science, Rehovot 76100, Israel, and \$Division of Solid State Physics/The Nanometer Structure Consortium, Lund University, Box 118, \\ S-221 00 Lund, Sweden
}

M etallic nanoantennas as a way to control the light emission of molecules or quantum dots have obtained more and more intense attention in recent years. ${ }^{1-8}$ Actually, the application of metal nanoparticle (NP) dimers as the simplest metallic nanoantennas to achieve single molecule detection by surfaceenhanced Raman scattering (SERS) was reported nearly a decade ago, ${ }^{9}$ although the term "nanoantennas" is seldom used in the SERS field. Metal nanoantennas close to emitters offer very efficient channels to couple the emitted photons to surface plasmons of the antenna, thus enhancing tremendously the efficiency of the emission. ${ }^{10}$ The photon energy coupled to the antennas may either be radiated again or become heat due to ohmic losses in metal structures. ${ }^{11}$ If the emitter is very close to the antenna (several nanometers), the spontaneous emission is mostly coupled to surface plasmons, rather than directly to free space. ${ }^{10}$ Therefore, the far-field emission of the emitter-antenna system is determined by the surface plasmon modes in the antenna, which can be used not only to enhance the emission of the emitter ${ }^{3-5,12,13}$ but also to control the emission direction ${ }^{6,14}$ and to guide light propagation even down to the single photon level. ${ }^{10}$

Recently, we have observed experimentally that the polarization of single molecule SERS signals from self-assembled silver NP trimers is dependent on the geometry and size of the NP aggregate. ${ }^{15}$ Theoretical simulation revealed that the interactions of light with NP trimers exhibiting broken symmetry resulting in wavelength-dependent rotation of the polarization of the emitted Raman signal. ${ }^{15} \mathrm{NP}$ trimers (or higher order structures) can
ABSTRACT We investigate the light emission from dipolar emitters located within nanoparticle antennas. It is found that the enormous emission enhancement can reach nearly a million fold. For multinanoparticle antennas, the polarization of the emissions strongly depends on the geometry of the antennas, the emitted wavelengths, and the dielectric functions of surrounding media. It is shown that a polarization nanorotator, which modulates the emission polarization on the nanometer scale, can be readily realized by varying either the geometry or surrounding media of nanoparticle antennas.

KEYWORDS: nanoparticle - polarization - nanoantenna - surface plasmon · enhancement

thus serve as modulators of the polarization of light on the nanoscale. However, it remains a challenging task to achieve systematic polarization control in this manner and to fully understand the mechanisms involved. Moreover, the exact enhancement of the emitted power in such a system is still not clear, although the emission enhancement of a molecule in SERS is generally accepted to be similar to the local intensity enhancement of $E^{2}$ (which can be shown by applying optical reciprocity ${ }^{15}$ ) with overall SERS enhancement scaled as $E^{4}$. Here we explore this issue further through a series of generalized Mie theory calculations, ${ }^{16}$ and we report that the polarization of the light emission can be easily changed either by moving one metal NP away from the symmetric axis of the NP linear array or by varying the surrounding dielectric medium. By integrating Poynting vectors over the closed surface containing multiparticle nanoantennas, the emitted power can be calculated exactly, which is found to be enhanced nearly one million fold.

In considerations below, NP nanoantennas are excited by a two-level emitter which can be treated as a dipole located at the cavity between NPs. The far-field emission of the dipole from NP antennas is calculated

\section{*Address correspondence to hxxu@iphy.ac.cn.}

Received for review December 30, 2008 and accepted February 09, 2009.

Published online February 17, 2009. 10.1021/nn800906c CCC: \$40.75 


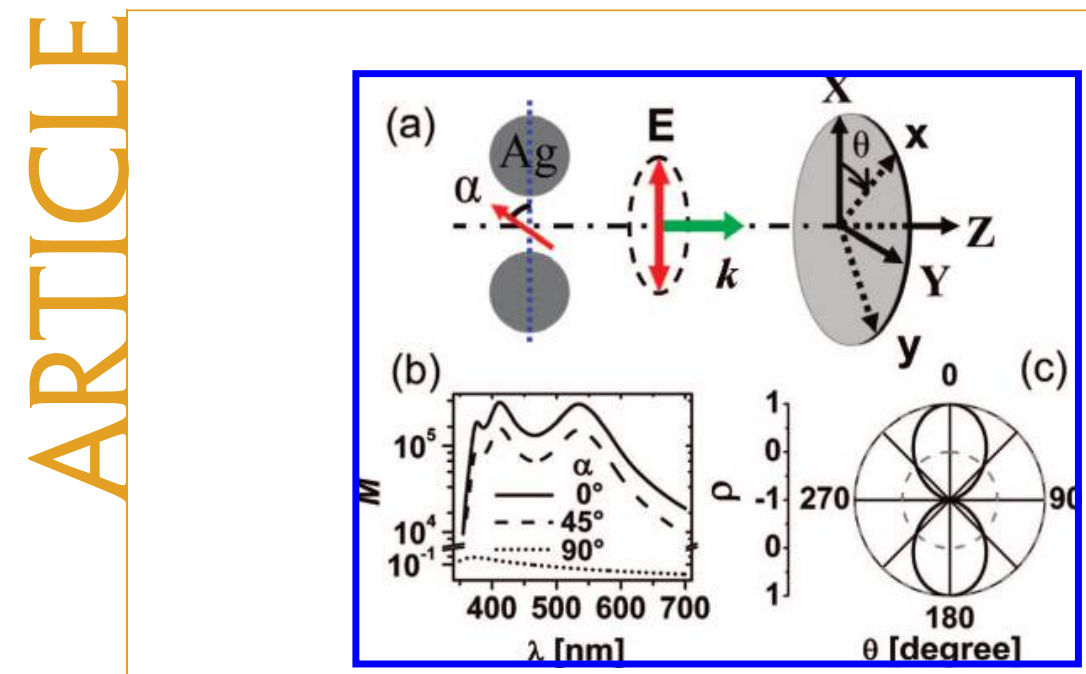

Figure 1. (a) Schematics of dipolar emission from a Ag dimer antenna. A dipole (red arrow) is located in the center between two silver NP $(R=40 \mathrm{~nm}$, separated by $1 \mathrm{~nm})$, with an orientation angle $\alpha$, and emits light at $555 \mathrm{~nm}$. The polarization of the emitted light is projected onto the $x$ and $y$ axes, and two orthogonal components of the emission are calculated for different rotation angles $\theta$ of the $x y$ axis with respect to the $X Y$ axis system to compute the depolarization ratio defined by formula 1. (b) Enhancement effect of dipole emission as a function of wavelength at different orientation angles $\alpha$. (c) Depolarization ratio of the emission from the dipole-dimer system. The refractivity of the surrounding medium is taken as $n_{\mathrm{s}}=1$.

by the generalized Mie theory. ${ }^{16,17}$ Two orthogonal components of the emission $I_{x}$ and $l_{y}$, projected on a rotating coordinate frame as illustrated in Figure $1 \mathrm{a}$, are computed. The depolarization ratio of the light emitted by the dipole through antennas can be obtained by

$$
\rho(\theta)=\frac{I_{x}(\theta)-I_{y}(\theta)}{I_{x}(\theta)+I_{y}(\theta)}
$$

The ellipticity of the emission is then $T=(1-$ $\left.\rho_{\max }\right) /\left(1+\rho_{\max }\right)$, where $\rho_{\max }$ is the maximum depolarization ratio. The angle $\theta_{\max }$ corresponding to $\rho_{\max }$ is defined as the direction of the polarization. The emission enhancement of the antenna can be calculated by integrating the Poynting vector over a surface $S$ enclosing the dipole-antenna system:

$$
M=\frac{\frac{1}{2} \operatorname{Re} \int_{S}\left(\mathbf{E}_{\text {dipole-antenna }} \times \mathbf{H}_{\text {dipole-antenna }}\right) \cdot \mathbf{n d s}}{\frac{1}{2} \operatorname{Re} \int_{S}\left(\mathbf{E}_{\text {dipole }} \times \mathbf{H}_{\text {dipole }}\right) \cdot \mathbf{n d s}}
$$

where the $\mathbf{E}$ and $\mathbf{H}$ in the numerator and denominator are electromagnetic fields emitted by the dipole with and without the NP antenna, respectively.

Dimer Case. We start with the case of the dimer antenna illustrated in Figure 1a. As shown in Figure 1b, the emission enhancement calculated by eq 2 is wavelength-dependent. ${ }^{18}$ Surprisingly, when the dipole is aligned with the dimer axis, the maximum enhancement factor, $M$, at $410 \mathrm{~nm}$ can reach $3.2 \times 10^{5}$. When the dipole is rotated away from the dimer axis, the enhancement decreases following the relation $\cos ^{2}$

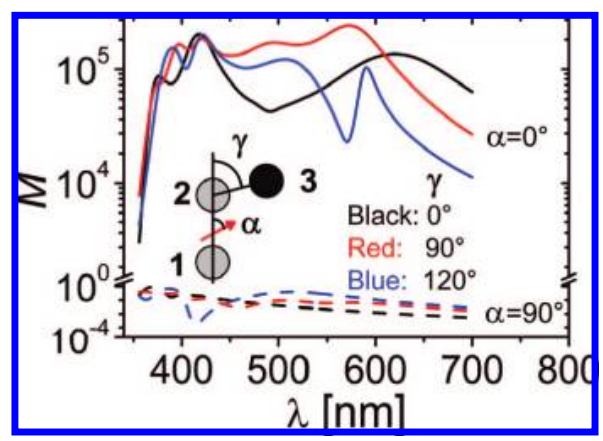

Figure 2. Enhancement factor $M$ of the dipole emission from trimer antenna as a function of the wavelength for different orientation angles $(\alpha)$ of the dipole (red arrow in the inset) and positions of the third NP. The black, red, and blue lines are for $\gamma=0,90$, and $120^{\circ}$, respectively. The solid and dashed lines are for the dipolar orientation $\alpha=0$ and $90^{\circ}$, respectively. The separations between $1 \mathrm{st}-2 \mathrm{nd}$ particles and $2 \mathrm{nd}-3 \mathrm{rd}$ particles are kept at $1 \mathrm{~nm}$. The refractivity of the surrounding medium is taken as $n_{\mathrm{s}}=1$.

$\alpha$. This shows that only the emission by the parallel component of the dipole can be enhanced significantly, while the perpendicular component is not enhanced. The antenna can enhance the dipole emission with $M$ $>10^{5}$ over a wide wavelength range from 370 to 600 $\mathrm{nm}$. Shown in Figure 1c, the calculated depolarization ratio of light emitted by the dipole-dimer system along the $Z$ axis reaches its maximum when $\theta=0^{\circ}$, which means that light emission from the dimer antenna is linearly polarized along the dimer axis. More calculations (Supporting Information) for the dipole with different orientation $\alpha$ show that this highly symmetric nanoantenna can only provide linearly polarized emission with the polarization parallel to the dimer axis.

Trimer Case. Another relatively simple type of nanoantenna is a NP trimer; that is, a third Ag nanoparticle ( $R$ $=40 \mathrm{~nm}$, the black sphere shown in the inset of Figure 2 ) is introduced into the dimer system. The plasmonic properties of some symmetric trimer systems have been studied..$^{19-21}$ Here, we maintain the configuration of the original dipole-dimer system, while adding a third NP which is kept adjacent to the second NP (separated from it by $1 \mathrm{~nm}$ ), while its position is being changed. The emission enhancement factors of trimer antennas as a function of wavelength are shown in Figure 2. For different geometries of the trimer, linear $(\gamma$ $=0^{\circ}$, black data), right angle $\left(\gamma=90^{\circ}\right.$, red), and equilateral triangle ( $\gamma=120^{\circ}$, blue), the position and shape of the resonant peak of the enhancement changes a lot due to the different surface plasmon couplings between the NPs. Importantly, as the dipolar orientation changes, $M$ still obeys the $\cos ^{2} \alpha$ rule over the whole wavelength range investigated, just as in the dimer case. It is obvious that only the emission from the parallel component of the dipole can be enhanced significantly, while no enhancement at all is obtained for the perpendicular component. For the dipole aligned to the dimer axis, the emission enhancement remains larger 
than $10^{4}$ over the wavelength range from $\sim 360$ to $\sim 650 \mathrm{~nm}$. A Fano-type profile arises at $572 \mathrm{~nm}$ for the equilateral triangle configuration of the trimer (solid blue curve). This originates from the interference of the dipolar subradiant and super-radiant modes of the coupled trimer as the third NP is rotated close to the first NP. 22,23

The depolarization ratio function $\rho(\theta)$ obtained for different geometries is shown in Figure 3. As the axial symmetry still holds (Figure 3a), the polarization of the emission from this linear trimer antenna is still along the symmetric axis, but when the third one is moved away from the symmetric axis, the polarization of the emission from the trimer is rotated to a new direction. As shown in Figure $3 \mathrm{~b}$, the emission from the trimer with right-angle geometry is polarized at an angle of $40^{\circ}$ relative to the symmetry axis of the dimer. When the third NP is moved to make a trimer with an equilateral triangle configuration as shown in Figure $3 c$, the polarization of the emission is rotated back again to be parallel to the axis of the original dimer since the third NP now couples symmetrically with the other two. Figure $3 d$ shows how the emission polarization is changed as the third NP rotates clockwise around the second NP. The polarization of the antenna emission first rotates with the same direction clockwise to $40^{\circ}$, the maximum polarization rotation. As the third NP rotates further, the emission polarization starts to rotate counterclockwise back to $0^{\circ}$. In parallel, the maximum of the depolarization ratio $\rho$ decreases to 0.7 (the ellipticity is $T=0.18$ ), then back to 1 , which means the emitted light is not fully linearly polarized but becomes slightly elliptical. What should be noted is that the depolarization ratio and the rotation angle are independent of the orientation of the dipole $\alpha$ (see Supporting Information). They only depend on the position of the third particle defined by the angle $\gamma$. This is yet again a manifestation of the fact that the depolarization ratio does not depend on the properties of the emitter, as shown in ref 15.

Coupling of the introduced third NP with the NP dimer modifies the symmetric surface plasmon modes of the dimer. When $\gamma$ is $0-90^{\circ}$, the third particle is mainly coupled with the second one. Hence, two strongly coupled surface plasmon modes contribute to the far-field emission: one due to $1 \mathrm{st}-2$ nd particle coupling and the other due to 2 nd-3rd particle coupling (the coupling of $1 \mathrm{st}-3 \mathrm{rd}$ particles is negligible). The former coupling, as discussed in the dimer case, determines the enhancement effect of the antenna, while the latter determines the rotation of the emission polarization. Different orientations of the third to the second NPs give rise to different couplings of surface plasmon modes, thus resulting in different angles of the polarization rotation. After $\gamma$ passes $90^{\circ}$, the third NP gets closer to the first one, so that the increased couplings between the third and

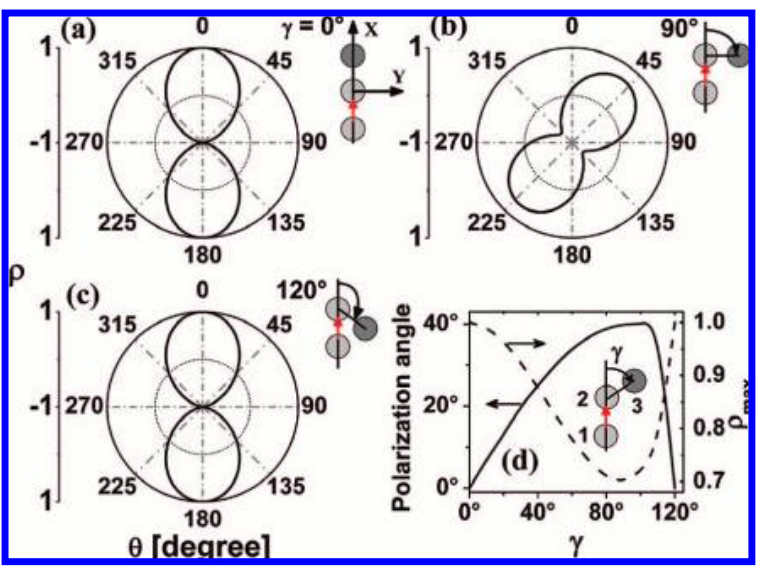

Figure 3. Depolarization ratio of the dipole emission from a Ag trimer antenna with (a) linear, (b) right-angle configurations, and (c) equilateral triangle. (d) Polarization angle (left axis) and the maximum depolarization ratio $\rho_{\max }$ (right axis) of the antenna emission as a function of angle $\gamma$. The radii of all three Ag NPs are identical $(R=40 \mathrm{~nm})$. The separations between $1 \mathrm{st}-2 \mathrm{nd}$ NPs and $2 \mathrm{nd}-3 \mathrm{rd}$ NPs are kept at $1 \mathrm{~nm}$. The wavelength of the dipole emission is $555 \mathrm{~nm}$. The refractivity of the surrounding medium is $n_{\mathrm{s}}=1$.

the first NPs lead to the polarization of the emission rotating rapidly back to the dimer case.

At different wavelengths of the dipole emission, the polarization rotation by similar trimer antennas could be very different. In Figure 4a, we investigate the dependence of the polarization angle of the emission from a right-angle trimer with a dipole emitting at a range of wavelengths from 450 to $650 \mathrm{~nm}$. This wavelength range covers the spectral range of quantum dots and dye molecules commonly used. Surprisingly, the polarization of the antenna emission excited by a dipole at the short wavelength part of this range rotates counterclockwise relative to the dimer axis, which is very different from the clockwise rotation seen for the long wavelength of the dipole emission. Near $520 \mathrm{~nm}$, there exists a sharp transition, where the polarization rotation shifts abruptly from -30 to $40^{\circ}$. This may be

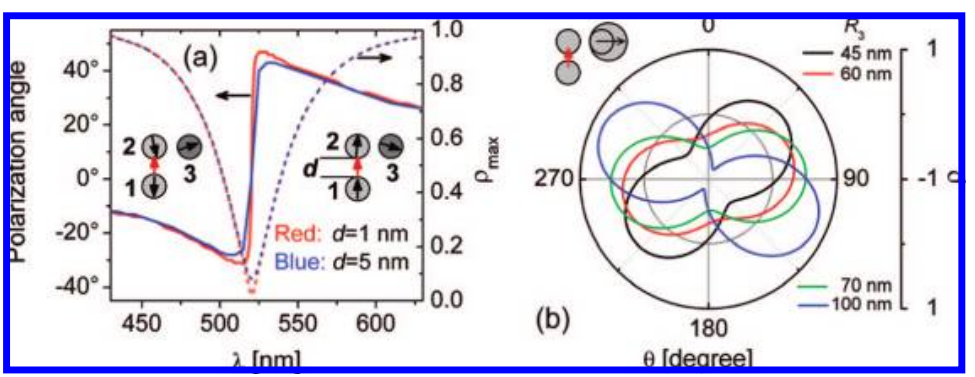

Figure 4. (a) Polarization angle (solid lines and left axis) and the maximum depolarization ratio $\rho_{\max }$ (dashed lines and right axis) as a function of the wavelength of the dipole emission for the right-angle configuration of a Ag trimer antenna with identical NPs $(R=40 \mathrm{~nm})$. The separation between the $1 \mathrm{st}$ and $2 \mathrm{nd}$ NPs is either $d=1 \mathrm{~nm}$ (red) or $d=5 \mathrm{~nm}$ (blue). The separation between the $2 \mathrm{nd}$ and 3rd NPs is always kept at $1 \mathrm{~nm}$. Black arrows in insets: representations of the dipolar polarization excited in each NP. (b) Depolarization ratio of light emitted from a right-angle $\mathrm{Ag}$ trimer with increasing radius of the third nanoparticle: $R_{1}=R_{2}=40 \mathrm{~nm}, R_{3}=45,60,70$, and $100 \mathrm{~nm}$. Separations between particles are kept at $1 \mathrm{~nm}$. The wavelength of the dipole emission is $555 \mathrm{~nm}$. The refractivity of the surrounding medium is $n_{s}=1$. 
caused by the plasmonic resonance between the second and third nanoparticles in the dipole-trimer system (not shown). For a shorter wavelength than this resonant wavelength, the plasmon excitation of the trimer has an opposite phase to the dipole, as illustrated in the inset (to the left), which is very different from the in-phase plasmonic excitation at the longer wavelength illustrated in the inset to the right. Interestingly, at the transition point, the emission from the trimer antenna is almost circularly polarized (the ellipticity is $T$ $=0.96$ ). When the separation between the first and the second NPs increases to $d=5 \mathrm{~nm}$ in order to hold a larger dipole emitter, the polarization rotation of the dipole emission by the antenna remains almost the same (blue curves in Figure 4a), but the emission enhancement of the antenna is largely reduced, roughly as (1/ d) ${ }^{2}$, which is similar to the electromagnetic field enhancement decrease as the distance from the surface increases. $^{24}$

If the size of the third particle is increased, the position of transition from negative to positive polarization rotation will red shift due to the retardation effect (Supporting Information). At the same time, a larger rotation of the emitted light can be achieved, as shown in Figure 4b. At the dipole emission wavelength $(\lambda=555$ $\mathrm{nm})$, the polarization direction is clockwise rotated. For the case of $R_{3}=70 \mathrm{~nm}$, the emission is almost $90^{\circ}$ polarized, with moderate ellipticity $T=0.5$. Even further rotation is achieved with $R_{3}=100 \mathrm{~nm}$. For a systematic exploration of this effect, see ref 15 .

Besides the position of the third NP, the refractivity of the surrounding medium, $n_{\mathrm{s}}$, can also tune the surface plasmon couplings in trimer antennas. As shown in Figure 5, the polarization rotation of the dipole emission from the right-angle trimer strongly depends on $n_{s}$. The multiple peaks in the spectrum of $M$ versus $n_{s}$ (Figure 2) imply the complexity of the way by which surface plasmon couplings determine the polarization of the far-field emission. There are several plasmon resonant peaks in the spectrum of the trimer antenna system due to the complex couplings between particles. For each of these resonances, there is a corresponding transition from counterclockwise to clockwise polarization rotation. When $n_{\mathrm{s}}$ is changed, the plasmon resonances will be also change, resulting in alternating counterclockwise and clockwise polarization rotations. Thus, the sensitivity of the emission polarization of the dipole-antenna system to $n_{\mathrm{s}}$ can have a large potential in applications aiming at controlling the polarization status of the dipole emission. For example, a trimer antenna with a quantum dot located within it can be embedded in an electro-optic material such as $\mathrm{LiNbO}_{3}$ or electric-field poled polymers; the polarization of the dipole emission can then be modulated by varying the applied voltage to change the refractivity of the electrooptic material. ${ }^{25}$ The emission enhancement is plotted as a function of the emission wavelength for several val-

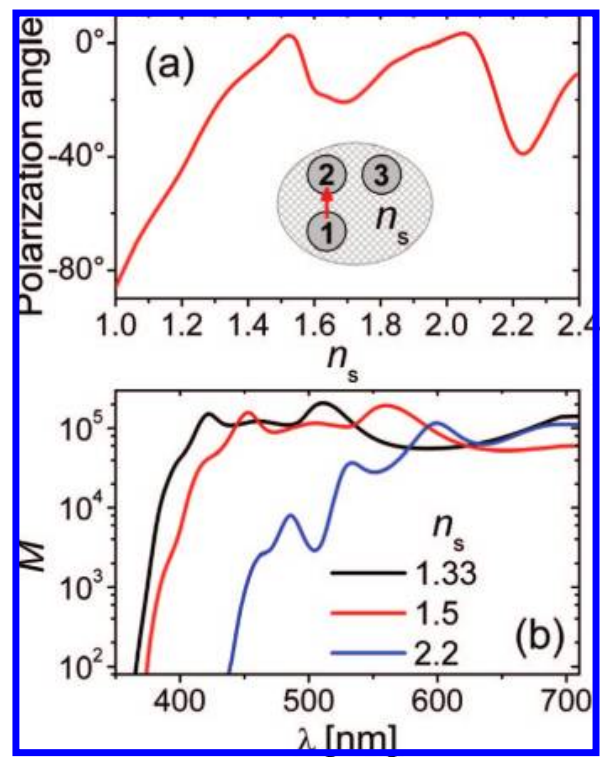

Figure 5. (a) Polarization angle of the emission from a rightangle trimer $\left(R_{1}=R_{2}=40 \mathrm{~nm}, R_{3}=80 \mathrm{~nm}\right)$ as a function of the refractivity of the surrounding medium $n_{s}$. The dipole, emitting at $555 \mathrm{~nm}$, is denoted by a red arrow. The separation between the 1 st and 2nd NPs is $d=1 \mathrm{~nm}$. The separation between the $2 \mathrm{nd}$ and $3 \mathrm{rd}$ NPs is kept at $1 \mathrm{~nm}$. (b) Emission enhancement from the same dipole-trimer system as a function of the emission wavelength of the dipole, for three different values of $n_{s}$.

ues of $n_{\mathrm{s}}$ in Figure $5 \mathrm{~b}$. It is clear that different $n_{\mathrm{s}}$ will change the position of the plasmon resonances of the antenna, but the largest enhancement is maintained at $\sim 10^{5}$ for a short separation $d=1 \mathrm{~nm}$ between the particles, even though it occurs at different resonant wavelengths.

Tetramer Case. A more efficient polarization rotator can be achieved by introducing a fourth NP, which is put at the other end of the dimer. Similar to the trimer case in Figure 3, the linear and equilateral triangle geometries do not rotate the polarization of the dipole emission (Figure 6a,c, respectively). When the third and the fourth NPs are positioned with a right/left-angle configuration (Figure $6 \mathrm{~b}$ ), the largest polarization rotation $\left(56^{\circ}\right)$ can be achieved. The plasmon coupling between the new particle and the trimer results in further clockwise rotation compared to the trimer case. As the third and fourth NPs are synchronously rotated counterclockwise around the center of second and first (Figure $6 d)$, the polarization of the emission from the tetramer antenna is clockwise-rotated up to a maximum obtained at $\gamma=90^{\circ}$, then rotated rapidly back to the symmetric axis of the dimer. At the transition point of the polarization rotation, the dipole emission by the antenna becomes slightly elliptical with an ellipticity of 0.25 .

Although all the considerations above are based on spherical particles, various kinds and shapes of NPs could be used to compose a polarization-rotating antenna, as well. For example, the wavelength range can be largely tuned to infrared by adopting core-shell NPs. ${ }^{26}$ More practically, a SPM tip (metal coated or NP 
embedded $)^{27-29}$ scanning over a NP dimer can function as a third particle in a trimer antenna. Nanoparticles could also be manipulated by an AFM tip. ${ }^{30,31}$ Recently, it was found that a single-photon source, such as CdSe quantum dot or nitrogen-vacancy color center, ${ }^{32}$ is able to excite single surface plasmons of $\mathrm{Ag}$ nanostructures. ${ }^{10,11}$ Hence, the trimer or tetramer antennas discussed above excited by these single-photon sources can serve as efficient and polarization controllable single-photon sources on the nanometer scale, which could have significant applications in the quantum cryptography ${ }^{33}$ and secure communication. ${ }^{34}$

\section{CONCLUSION}

In conclusion, we have investigated the properties of the dipole emission from Ag NP antennas. It was found that the polarization of light emission is determined by the surface plasmon modes of NP antennas, and the emission enhancement can reach nearly a million times. For the strong coupled metal NP dimer, the antenna only enhances the component of the dipole that is parallel to the dimer axis, which also results in a parallel polarized emission in the far field. The plasmon couplings between neighboring metal NPs, whose junction holds the dipole emitter, determine the enhancement of dipole emission by the antenna. The enhancement factor is strongly dependent on the separation between the NPs. When a third particle is added to form a trimer antenna, the polarization and depolarization ratio of the emission become geometry- and wavelengthdependent. The plasmon couplings between the third particle and the other two affect the polarization and ellipticity of the far-field emission. Indeed, the rightangle trimer antenna can rotate the far-field polarization by $\pm 40^{\circ}$ with respect to the dimer axis. A larger rotation angle $\left( \pm 56^{\circ}\right)$ can be obtained in a tetramer antenna. By increasing the size of the third or fourth nano-

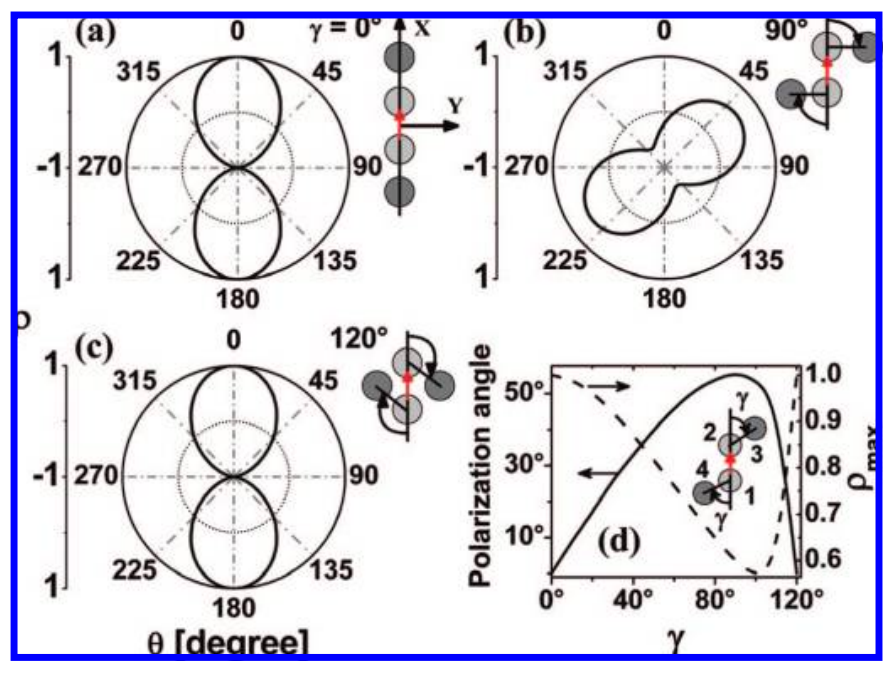

Figure 6. Emission polarization of a Ag tetramer antenna. $(a-c)$ Depolarization ratio as a function of $\boldsymbol{\theta}$, for the linear, right angle, and equilateral triangle aligned tetramer, respectively. (d) Polarization angle (to the left) and the maximum depolarization ratio $\rho_{\max }$ (to the right) of the antenna emission as a function of the rotation of the third and fourth NP around the center of the second NP and the first one, respectively. The radii of Ag NPs are all identical $(R=40 \mathrm{~nm})$. The separations between the first and second, second and third, first and fourth NPs are kept at $1 \mathrm{~nm}$. The wavelength of the dipole emission is $555 \mathrm{~nm}$. The refractivity of the surrounding medium is $n_{\mathrm{s}}=1$.

particle, the far-field can be even $90^{\circ}$ polarized. We also found that the polarization rotation of the nanoantenna is highly sensitive to the refractivity of the surrounding medium. This property can be used to make either dielectric tunable polarization nanorotators or surface-plasmon-based refractivity sensors. By adopting a single-photon emitter, such as a CdSe quantum dot or a color center, ${ }^{32,35}$ the proposed nanoantennas can be used to control the polarization of a singlephoton source. Hence, promising applications could be envisioned in such emerging fields as quantum cryptography $^{33}$ and integrated optics. ${ }^{36,37}$

\section{CALCULATION METHOD}

On the basis of the generalized Mie theory, ${ }^{16,17}$ the incident and scattered electromagnetic fields are expanded into vector spherical harmonics (VSH). The expansion coefficients for the incident field are well-known as Mie coefficients. ${ }^{38}$ The scattered electric fields for $\mathrm{N}$-spheres system are

$$
E_{S}=\sum_{l=1}^{\oplus N} E_{S}^{l}=\sum_{l=1}^{\oplus N} T_{i}^{\prime} W^{E}
$$

where ${ }^{\prime} W^{E}$ is the matrix of $\mathrm{VSH}^{N}{ }^{N} T_{n}$ is the scattering matrix for the $\mathrm{N}$-spheres system:

$$
\left[{ }^{N} T_{1},{ }^{N} T_{2},{ }^{N} T_{3}, \cdots{ }^{N} T_{N}\right]=\left[G_{1}, G_{2}, G_{3} \cdots G_{N}\right] \Psi^{(N)}
$$

$G_{N}$ is the incident coefficient for $N^{\text {th }}$ sphere, $\Psi^{(N)}$ is called the response matrix of the system, which is given below concisely (for details, see refs 16 and 17):

$$
\Psi_{N N}^{(N)}=S_{n_{i=0}}^{N o S}\left(\Omega^{(N-1)} \Psi^{(N-1)} \Omega \prime^{(N-1)} S_{n}\right)^{i}
$$

www.acsnano.org

$$
\begin{gathered}
\Psi_{p N}^{(N)}=\sum_{j=1}^{N-1} \Psi_{p j}^{(N-1)} \Omega_{j, N} \Psi_{N N}^{(N)} \quad p=1 \cdots N-1 \\
\Psi_{N q}^{(N)}=\Psi_{N N}^{(N)} \sum_{j=1}^{N-1} \Omega_{N, j} \Psi_{j q}^{(N-1)} \quad q=1 \cdots N-1 \\
\Psi_{p q}^{(N)}=\Psi_{p q}^{(N-1)}+\Psi_{p N}^{(N)} \sum_{j=1}^{N-1} \Omega_{N, j} \Psi_{j q}^{(N-1)} \quad p, q=1 \cdots N-1
\end{gathered}
$$

where $S_{n}$ is the scattering matrix for $n^{\text {th }}$ single sphere, $\Omega^{(N-1)}=$ $\left[\Omega_{N, 1} \Omega_{N, 2} \cdots \Omega_{N, N-1}\right], \Omega_{l, s}$ is the translation matrix from coordinates of the $I^{\text {th }}$ sphere to the $s^{\text {th }}$ sphere obtained by the addition theorem..$^{39}$ The permittivity of the Ag core is the value from the work of Johnson and Christy. ${ }^{40}$

For a dipole at the point $A$, the expansion coefficient is

$$
G_{\mathrm{A}}=\left[0 \cdots 0 C 2_{-11}^{\mathrm{A}} C 2_{-11}^{\mathrm{A}} C 2_{11}^{\mathrm{A}}\right]
$$

The incident coefficients for $t^{\text {th }}$ sphere is expressed as

$$
G_{l}=G_{A} \Omega_{A l}
$$


Acknowledgment. H.X. was supported by National Natural Science Foundation of China under Contract No. 10625418, by MOST under Contract Nos. 2006DFB02020, 2007CB936800, and 2009CB930704, and by the "Bairen" projects of CAS. G.H. was supported in part by the Israel Science Foundation (Grant No. 958/06).

Supporting Information Available: The polarization angle at different dipole orientation and the wavelength dependence of polarization angle. This material is available free of charge via the Internet at http://pubs.acs.org.

\section{REFERENCES AND NOTES}

1. Curto, A. G.; García de Abajo, F. J. Near-Field Optical Phase Antennas for Long-Range Plasmon Coupling. Nano Lett. 2008, 8, 2479-2484.

2. Mühlschlegel, P.; Eisler, H. J.; Martin, O. J. F.; Hecht, B.; Pohl, D. W. Resonant Optical Antennas. Science 2005, 308, 1607-1609.

3. Farahani, J. N.; Pohl, D. W.; Eisler, H. J.; Hecht, B. Single Quantum Dot Coupled to a Scanning Optical Antenna: A Tunable Superemitter. Phys. Rev. Lett. 2005, 95, 017402.

4. Kuhn, S.; Hakanson, U.; Rogobete, L.; Sandoghdar, V. Enhancement of Single-Molecule Fluorescence Using a Gold Nanoparticle as an Optical Nanoantenna. Phys. Rev. Lett. 2006, 97, 017402.

5. Taminiau, T. H.; Stefani, F. D.; Segerink, F. B.; Van Hulst, N. F. Optical Antennas Direct Single-Molecule Emission. Nat. Photon. 2008, 2, 234-237.

6. Taminiau, T. H.; Moerland, R. J.; Segerink, F. B.; Kuipers, L.; van Hulst, N. F. lambda/4 Resonance of an Optical Monopole Antenna Probed by Single Molecule Fluorescence. Nano Lett. 2007, 7, 28-33.

7. Tang, L.; Kocabas, S. E.; Latif, S.; Okyay, A. K.; Ly-Gagnon, D. S.; Saraswat, K. C.; Miller, D. A. B. Nanometre-Scale Germanium Photodetector Enhanced by a Near-Infrared Dipole Antenna. Nat. Photon. 2008, 2, 226-229.

8. Bryant, G. W.; De Abajo, F. J. G.; Aizpurua, J. Mapping the Plasmon Resonances of Metallic Nanoantennas. Nano Lett. 2008, 8, 631-636.

9. Xu, H. X.; Bjerneld, E. J.; Käll, M.; Börjesson, L. Spectroscopy of Single Hemoglobin Molecules by Surface Enhanced Ranman Scattering. Phys. Rev. Lett. 1999, 83, 4357-4360.

10. Akimov, A. V.; Mukherjee, A.; Yu, C. L.; Chang, D. E.; Zibrov, A. S.; Hemmer, P. R.; Park, H.; Lukin, M. D. Generation of Single Optical Plasmons in Metallic Nanowires Coupled to Quantum Dots. Nature 2007, 450, 402-406.

11. Chang, D. E.; Sorensen, A. S.; Demler, E. A.; Lukin, M. D. A Single-Photon Transistor Using Nanoscale Surface Plasmons. Nat. Phys. 2007, 3, 807-812.

12. Johansson, $\mathrm{P} ; \mathrm{Xu}, \mathrm{H}$. X.; Kall, M. Surface-Enhanced Raman Scattering and Fluorescence Near Metal Nanoparticles. Phys. Rev. B 2005, 72, 17.

13. Muskens, O. L.; Giannini, V.; Sanchez-Gil, J. A.; Rivast, J. G. Strong Enhancement of the Radiative Decay Rate of Emitters by Single Plasmonic Nanoantennas. Nano Lett. 2007, 7, 2871-2875.

14. Knight, M. W.; Grady, N. K.; Bardhan, R.; Hao, F.; Nordlander, P.; Halas, N. J. Nanoparticle-Mediated Coupling of Light into a Nanowire. Nano Lett. 2007, 7, 2346-2350.

15. Shegai, T.; Li, Z.; Dadosh, T.; Zhang, Z.; Xu, H.; Haran, G. Managing Light Polarization via Plasmon-Molecule Interactions within an Asymmetric Metal Nanoparticle Trimer. Proc. Natl. Acad. Sci. U.S.A. 2008, 105, 16448-16453.

16. Li, Z. P.; Xu, H. X. Electromagnetic Energy Flow Near Metal Nanoparticles-II: Algorithms for the Calculation of the Light Scattering of Multi-Spheres and Photon Energy Transport via Linear Chains of Ag Nanoparticles. J. Quant. Spectrosc. Radiat. Transfer 2007, 103, 394-401.

17. Xu, H. X. A New Method by Extending Mie Theory to Calculate Local Field in Outside/Inside of Aggregates of Arbitrary Spheres. Phys. Lett. A 2003, 312, 411-419.

18. Lassiter, J. B.; Aizpurua, J.; Hernandez, L. I.; Brandl, D. W.; Romero, I.; Lal, S.; Hafner, J. H.; Nordlander, P.; Halas, N. J.
Close Encounters between Two Nanoshells. Nano Lett. 2008, 8, 1212-1218.

19. Brandl, D. W.; Mirin, N. A.; Nordlander, P. Plasmon Modes of Nanosphere Trimers and Quadrumers. J. Phys. Chem. B 2006, 110, 12302-12310.

20. Alegret, J.; Rindzevicius, T.; Pakizeh, T.; Alaverdyan, Y.; Gunnarsson, L.; Kall, M. Plasmonic Properties of Silver Trimers with Trigonal Symmetry Fabricated by ElectronBeam Lithography. J. Phys. Chem. C 2008, 112, 14313-14317.

21. Giannini, V.; Sanchez-Gil, J. A. Excitation and Emission Enhancement of Single Molecule Fluorescence through Multiple Surface-Plasmon Resonances on Metal Trimer Nanoantennas. Opt. Lett. 2008, 33, 899-901.

22. Le, F.; Brandl, D. W.; Urzhumov, Y. A.; Wang, H.; Kundu, J.; Halas, N. J.; Aizpurua, J.; Nordlander, P. Metallic Nanoparticle Arrays: A Common Substrate for Both Surface-Enhanced Raman Scattering and SurfaceEnhanced Infrared Absorption. Acs Nano 2008, 2, 707-718.

23. Christ, A.; Ekinci, Y.; Solak, H. H.; Gippius, N. A.; Tikhodeev, S. G.; Martin, O. J. F. Controlling the Fano Interference in a Plasmonic Lattice. Phys. Rev. B 2007, 76, 201405R.

24. Xu, H. X.; Aizpurua, J.; Käll, M.; Apell, P. Electromagnetic Contributions to Single-Molecule Sensitivity in SurfaceEnhanced Raman Scattering. Phys. Rev. E 2000, 62, 4318 4324.

25. Yariv, A. Optical Electronics; Saunders College: Philadelphia, 1991

26. Brandl, D. W.; Oubre, C.; Nordlander, P. Plasmon Hybridization in Nanoshell Dimers. J. Chem. Phys. 2005, $123,024701$.

27. Hartschuh, A.; Sanchez, E. J.; Xie, X. S.; Novotny, L. HighResolution Near-Field Raman Microscopy of Single-Walled Carbon Nanotubes. Phys. Rev. Lett. 2003, 90, 095503.

28. Olk, P.; Renger, J.; Hartling, T.; Wenzel, M. T.; Eng, L. M. Two Particle Enhanced Nano Raman Microscopy and Spectroscopy. Nano Lett. 2007, 7, 1736-1740.

29. Olk, P.; Renger, J.; Wenzel, M. T.; Eng, L. M. Distance Dependent Spectral Tuning of Two Coupled Metal Nanoparticles. Nano Lett. 2008, 8, 1174-1178.

30. Tong, L. M.; Li, Z. P.; Zhu, T.; Xu, H. X.; Liu, Z. F. Single GoldNanoparticle-Enhanced Raman Scattering of Individual Single-Walled Carbon Nanotubes via Atomic Force Microscope Manipulation. J. Phys. Chem. C 2008, 112, 7119-7123.

31. Bek, A.; Jansen, R.; Ringler, M.; Mayilo, S.; Klar, T. A.; Feldmann, J. Fluorescence Enhancement in Hot Spots of AFM-Designed Gold Nanoparticle Sandwiches. Nano Lett. 2008, 8, 485-490.

32. Beveratos, A.; Kuhn, S.; Brouri, R.; Gacoin, T.; Poizat, J. P.; Grangier, P. Room Temperature Stable Single-Photon Source. Eur. Phys. J.D 2002, 18, 191-196.

33. Gisin, N.; Ribordy, G. G.; Tittel, W.; Zbinden, H. Quantum Cryptography. Rev. Mod. Phys. 2002, 74, 145-195.

34. Bennett, C. H.; Brassard, G. In Quantum Crytography: Public Key Distribution and Coin Tossing, Proceedings of the IEEE ICCSS; IEEE: New York, 1984; pp 175-179.

35. Wu, E.; Rabeau, J. R.; Roger, G.; Treussart, F.; Zeng, H.; Grangier, P.; Prawer, S.; Roch, J. F. Room Temperature Triggered Single-Photon Source in the Near Infrared. New J. Phys. 2007, 9, 434.

36. Quinten, M.; Leitner, A.; Krenn, J. R.; Aussenegg, F. R. Electromagnetic Energy Transport via Linear Chains of Silver Nanoparticles. Opt. Lett. 1998, 23, 1331-1333.

37. Maier, S. A.; Kik, P. G.; Atwater, H. A.; Meltzer, S.; Harel, E.; Koel, B. E.; Requicha, A. G. Local Detection of Electromagnetic Energy Transport Below the Diffraction Limit in Metal Nanoparticle Plasmon Waveguides. Nat. Mater. 2003, 2, 229-232.

38. Stratton, J. A. Electromagnetic Theory; McGraw-Hill: New York, 1941.

39. Stein, S. Addition Theorems for Spherical Wave Functions. Q. Appl. Math. 1961, 19, 15-24.

40. Johnson, P. B.; Christy, R. W. Optical Constants of the Noble Metals. Phys. Rev. B 1972, 6, 4370-4379. 